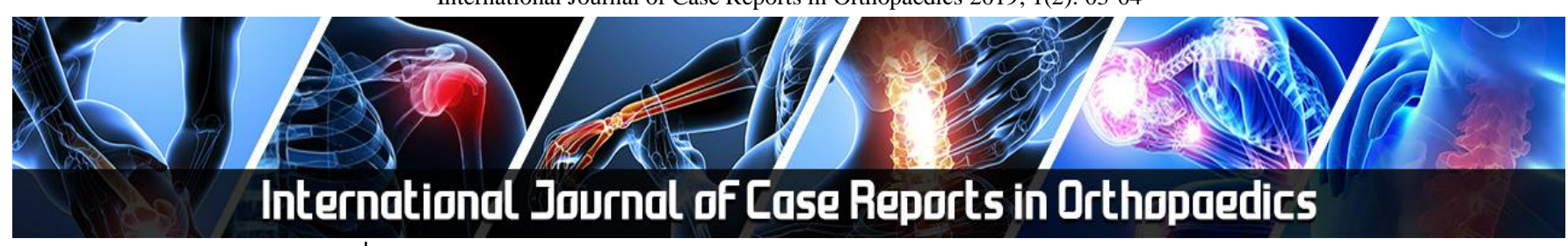

E-ISSN: 2707-8353 P-ISSN: 2707-8345 IJCRO 2019; 1(2): 03-04 Received: 05-05-2019 Accepted: 08-06-2019

Suhaib Ahmed Khan Department of Orthopaedics, Institute of Orthopaedics \& Surgery, Karachi, Pakistan
Corresponding Author: Suhaib Ahmed Khan Department of Orthopaedics, Institute of Orthopaedics \& Surgery, Karachi, Pakistan

\section{Diaphyseal Forearm fracture in 38 years old male patient- A case report}

\section{Suhaib Ahmed Khan}

DOI: https://doi.org/10.22271/27078345.2019.v1.i2a.7

\begin{abstract}
Diaphyseal Forearm fractures in adults are relatively common. Forearm fractures are regarded as articular fractures as slight deviation in the spatial orientation of the radius and ulna. Locked intramedullary nailing technique is the capacity of preventing shortening in metaphyseal, comminuted, and segmental diaphyseal forearm fractures. We recorded a diaphyseal forearm fracture in 38 years old male patient that was treated with intramedullary nailing.
\end{abstract}

Keywords: Diaphyseal, Forearm fracture, Intramedullary nailing

\section{Introduction}

Diaphyseal Forearm fractures in adults are relatively common. Forearm fracture in adult is break or crack that may occur near lower part of the forearm, near the wrist (distal region), center of the forearm (middle), at the upper part of the forearm, or near elbow (proximal region). Forearm bone fractures are also commonly encountered in today's industrial era. Fractures of both radius and ulna are one of the most common fractures in adults in upper extremity In this era of active life, rapid industrialisation, increasing road traffic accidents, competitive sports; the incidence of fractures of forearm bones are increasing in frequency ${ }^{[1]}$. Forearm fractures are regarded as articular fractures as slight deviation in the spatial orientation of the radius and ulna significantly decreases the forearm's rotational amplitude and thereby impairs the positioning and function of the hand. Patient with forearm fracture usually complain of pain and swelling depending upon the site involved [2]. Various treatment modalities were introduced from time to time and each of them had some edge over the previous one. Undisplaced fracture can be safely treated with cast or splint at the time of hospital admission. For displaced fracture casting under local anesthesia can be done. Fractures of the forearm bones may result in severe loss of function unless adequately treated. Severe loss of function of the radius and ulna present specific problems in addition to the problems common to all fractures of the shafts of long bones. In addition to regaining length, apposition, and axial alignment, achieving normal rotational alignment is necessary if a good range of pronation and supination is to be restored ${ }^{[3]}$.

Locked intramedullary nailing technique is the capacity of preventing shortening in metaphyseal, comminuted, and segmental diaphyseal forearm fractures, but the procedure is technically demanding and injury to posterior interosseous nerve is reported. Unlocked closed intramedullary nailing respects the soft tissues and vascular supply compared with open reduction. Sage described improved results with prebent triangular design ${ }^{[4]}$. We recorded a diaphyseal forearm fracture in 38 years old male patient that was treated with intramedullary nailing.

\section{Case report}

A 38 years old male visited the orthopaedic department with severe pain in lower forearm region since 1 day. History revealed that patient met with accident 1 day ago.

His physical examination was normal. All vitals were in normal limit. On examination, the right forearm region was painful with swelling. Patient was subjected to AP and lateral diaphyseal radiograph. Patient was also subjected to CT scan using Toshiba 6500 HT machine.

Both radiographs and $\mathrm{CT}$ scan revealed a displaced and angulated trifocal fracture of the radial shaft in combination with a displaced two-part mid-shaft ulna fracture. The nailing procedure was performed under brachial block in a supine position with the arm on side table. A tourniquet was applied to the upper arm and was inflated only if an open reduction of the fracture was necessary. A Nick with 11 no surgical blade from entry hole was made in 
the skin through lister's tubercle. A blunt dissection was made to avoid superficial branch of radial nerve and extensor tendons. An awl was used to make the entry hole in the radius. An oblique hole was made in the dorsal radius with a drill bit $4.5 \mathrm{~mm}$ and only one cortex was drilled. The hole was widened gently by rotating the drill bit and lowering it so that it came near parallel to the shaft. The bent tip of nail was used to aid the reduction and the nail was passed into the proximal radius upto the level of bicipital tuberosity. The nail was cut close to the bone, leaving enough length to allow for later removal but not so prominent that the skin was tented. The skin was closed over the tip.

For the ulna the entry hole was made at the tip of the olecranon process and nail was passed antegradely across fracture. The above elbow POP splint or cast was given and was removed 3 weeks after operation. Antibiotics are given IV for the first 5 days and then replaced with oral antibiotics till the 12th day after the surgery. Anti-inflammatory agents, analgesics and other supplements were given. Patient was on regular follow up. The prognosis of the patient was good.

\section{Discussion}

Fractures of both radius and ulna are one of the common fractures in adults in upper extremity. Healing occurs relatively after closed treatment but mal-union with resultant decreased rotation of the forearm is common and has been associated with poop outcomes. Loss of rotation impedes the function of the upper limb and activities of daily living. The treatment of displaced fractures of shafts of radius and ulna is primarily operative. The closed reduction and cast immobilization for the displaced fractures should only be taken if there is a specific contraindication to operative Treatment ${ }^{[5]}$.

Unlocked closed intramedullary nailing respects the soft tissues and vascular supply compared with open reduction. Unlocked nail may not adequately control rotation, especially in segmental fractures. The distinct advantage of locked intramedullary nailing technique is the capacity of preventing shortening in metaphyseal, comminuted, and segmental diaphyseal forearm fractures, but the procedure is technically demanding and injury to posterior interosseous nerve is reported ${ }^{[6]}$. We recorded a diaphyseal forearm fracture in 38 years old male patient that was treated with intramedullary nailing.

Hidaka et al. ${ }^{[7]}$ reported a case of trifocal, diaphyseal fracture of the radius with a mid-diaphyseal fracture of the ulna in a 53-year-old right-hand dominant woman involved in a head-on collision with another vehicle. Hong and colleagues ${ }^{[8]}$ described 32 fractures treated in 18 patients with interlocking intramedullary nail fixation. Eight of the patients had open fractures, but none required bone grafting. All patients were immobilized for at least 2 to 3 weeks in a splint or long arm cast. All fractures healed, and the average DASH score was 19 (range, 4 -72). Complications included 1 synostosis in a patient with a head injury, 4 superficial infections in patients treated with open reduction, and 2 patients in whom the distal interlocking screws in the ulna loosened, requiring removal.

Gadegone et al. ${ }^{[9]}$ in their study included seventy-six adults with forearm fractures (radius and ulna or isolated fracture of the single bone) were retrospectively evaluated. Fifty males and 26 females with the mean age of 38 years (range, 18-70 years) underwent closed reduction and screw intramedullary nail fixation. Ten patients required limited open reduction. The fractures were classified according to the AO/OTA system. The average follow up was 12 months (range, 6 to 18 months). The mean surgical time was 45 minutes (35 to 65 minutes). The meantime to union was 14 weeks (10-21 weeks). The results were graded as excellent in 50, good in 18 patients, and acceptable in eight patients, using the criteria of Grace and Eversman. Authors had superficial infection in three cases, one case of delayed infection, painful bursa in two cases, delayed union in two cases, malunion with dislocation of the DRUJ in two cases, injury to the extensor tendon of the thumb in one case, and one case of incomplete radioulnar synostosis.

Talwalkar et al. ${ }^{[10]}$ described 53 patients with 78 diaphyseal forearm fractures treated with a similar "minimum" screw technique, using fewer than 6 cortices of fixation on at least 1 side of the fracture. Ninety-one percent of the fractures united, with all non-unions occurring in open fractures with associated bone loss. There was 1 immediate hardware failure due to inadequate plate length with only 2 holes on 1 side of the fracture and 1 late hardware failure in which both screws on 1 side of the fracture broke.

\section{Conclusion}

Authors suggested diaphyseal forearm fractures

\section{References}

1. Visńa P, Beitl E, Pilný J, Cizmár I, Vlcek M, Kalvach J et al. Interlocking nailing of forearm fractures. Acta Chir Belg. 2008; 108:333-8.

2. Ozkaya U, Kiliç A, Ozdoğan U, Beng K, Kabukçuoğlu Y. Comparison between locked intramedullary nailing and plate osteosynthesis in the management of adult forearm fractures. Acta Orthop Traumatol Turc. 2009; 43:14-20.

3. Weckbach A, Blattert TR, Weisser CH. Interlocking nailing of forearm fractures. Arch Orthop Trauma Surg 2006; 126:309-15.

4. Tabor OB Jr, Bosse MJ, Sims SH, Kellam JF. Iatrogenic posterior interosseous nerve injury: Is transosseous static locked nailing of the radius feasible? J Orthop Trauma. 1995; 9:427-9.

5. Lee YH, Lee SK, Chung MS, Baek GH, Gong HS, Kim $\mathrm{KH}$. Interlocking contoured intramedullary nail fixation for selected diaphyseal fractures of the forearmin adults. J Bone Joint Surg Am. 2008; 90:1891-8.

6. Rosson JW. Refracture after the removal of plates from the forearm. An avoidable complication. Bone \& Joint Journal. 1991; 73(3):415-7.

7. Hidaka S, Gustilo RB. Refracture of bones of the forearm after plate removal. JBJS. 1984; 66(8):1241-3.

8. Hong G, Cong-Feng L, Chang-Qing Z, Hui-Peng S, Cun-Yi F, Bing-Fang Z. Internal fixation of diaphyseal fractures of the forearm by interlocking intramedullary nail: short-term results in eighteen patients. Journal of orthopaedic trauma. 2005; 19(6):384-91.

9. Gadegone W, Salphale YS, Lokhande V. Screw elastic intramedullary nail for the management of adult forearm fractures. Indian J Orthop. 2012; 46:65-70.

10. Talwalkar AK, Talwalkar CA. Internal fixation of fractures of redius and ulna in adults with Talwalkar intramedullary nails. Indian J Orthop. 1967; 1(1):26-30. 